\title{
Gyre Precoding for MU-MIMO Systems
}

\author{
J.C. De Luna Ducoing, Member, IEEE, Yiping Qin, Yun Xue, \\ and Konstantinos Nikitopoulos Senior Member, IEEE
}

\begin{abstract}
This work introduces Gyre Precoding (GP), a novel linear multi-user multiple-input multiple-output (MU-MIMO) precoding approach. GP performs rotations of the symbols of each spatial layer to optimize the precoding performance. To find the rotation angles, we propose a near-optimal, gradient descent-based low-complexity algorithm. GP is constellationagnostic and does not require significant changes to conventional receiver procedures or wireless standards. Computer evaluation results show that GP can achieve $8 \mathrm{~dB}$ SNR gains over linear precoding techniques and $2 \mathrm{~dB}$ over suboptimal symbol-level precoding (SLP) methods for a $16 \times 16$ MU-MIMO system. Furthermore, in a $64 \times 12$ massive-MIMO scenario in a $5 \mathrm{G}$ New Radio (5GNR) setup, GP achieves a 13\% higher throughput gain over zero-forcing precoding.
\end{abstract}

Index Terms-Multi-user multiple-input multiple-output (MUMIMO), precoding.

\section{INTRODUCTION}

$\mathbf{M}$ ULTI-USER multiple-input multiple-output (MUMIMO) precoding can achieve a high sum spectral efficiency by making use of the spatial domain to serve multiple users (UEs). Several precoding techniques that offer near-optimal performance have been proposed. However, many suffer from a high complexity or require specialized receiverside processing. Therefore, simple and low-complexity precoding techniques that do not require significant changes to conventional transceiver procedures or current wireless standards are of high value.

The simplest precoding methods are linear techniques [1], where the design of a precoding filter depends only on the MIMO propagation channel. Commonly-used approaches are zero forcing (ZF) and linear minimum mean square error (MMSE). Linear techniques exhibit a low complexity and have the advantage that the precoder is calculated only once for each channel realization. However, they suffer from a substantial SNR loss when the MIMO channel is ill-conditioned.

Other precoder techniques use linear filters as a baseline but improve them by performing additional signal processing based on the characteristics of the data symbols. Among these are the non-linear perturbation-based approaches, where the most common technique is vector perturbation (VP) [2]. VP processing involves solving an integer least squares (ILS) cost function at every transmission. The optimal solution can be found using a sphere encoder [2], but at a substantial expense in computational complexity. Some approaches make use of massive parallelization to deal with the complexity and offer

J.C. De Luna Ducoing and Konstantinos Nikitopoulos are with the 5G and 6G Innovation Centre, Institute for Communication Systems, University of Surrey, Guildford, UK, GU2 7XH. (e-mail: jd0050@surrey.ac.uk, k.nikitopoulos@surrey.ac.uk). Yiping Qin and Yun Xue are with Shanghai Huawei Technologies Co., Ltd. (e-mail: qinyiping@huawei.com, xueyun4@huawei.com). a reduced processing latency [3]. However, modulo operations or specialized log-likelihood (LLR) calculations are required to remove the effects of the perturbed symbols. Therefore, the use of perturbation-based precoding would require significant changes to conventional receivers and wireless standards.

Symbol-level precoding (SLP)-based methods are another type of non-linear precoding techniques that take the data into account to calculate the precoder. These discern between constructive and destructive multi-user interference and aim to exploit the former and suppress the latter. SLP methods increase the SINR by relaxing the optimization procedure to allow some symbols to lie within the constructive interference regions (CIR) of constellations [4]. The optimal SLP solution can be found using quadratic optimization [5], but suffers from high complexity. Suboptimal solutions include the ones proposed in [4], [5], [6]. SLP techniques do not require major changes to conventional receiver processing. However, for SLP methods, the design of the precoder and its performance are highly dependent on the constellations used. Moreover, the precoder needs to be computed at every timeslot.

In this paper, we propose a simple and linear alternative approach to MU-MIMO precoding based on the rotation of the data symbols, which we denote as Gyre Precoding (GP). In comparison to perturbation-based approaches, GP does not require significant changes to conventional receiver procedures or wireless communication standards, and in contrast to SLP techniques, it is modulation-agnostic. Similar to these nonlinear precoding methods, the precoder in GP is based on the characteristics of the data, but it is calculated once per frame. GP performs gyrations of each data stream, with the same rotation angle applied to all symbols in a frame. Precoded pilots are used to convey the angle information to the receivers, which use it to de-rotate the symbols before demodulation. The rotation angles are chosen such that they minimize the quadratic GP objective function. Since a closed-form optimal solution is difficult, we propose a gradient descent-based algorithm that finds the near-optimal solution with a low expense in complexity.

GP offers a power gain dependent on the channel matrix condition number and the frame length. We show that GP achieves a gain of approximately $8 \mathrm{~dB}$ over MMSE precoding, with a small increase in complexity, and $2 \mathrm{~dB}$ over the SLP method in [5], with lower complexity, for an MU-MIMO system with 16 base station (BS) antennas and 16 single-antenna UEs. Additionally, an evaluation of a $64 \times 12$ massive-MIMO scenario in a $5 \mathrm{G}$ New Radio (5GNR) setting verifies that GP offers $13 \%$ higher throughput gains over conventional linear techniques when practical channel estimation procedures are used. 


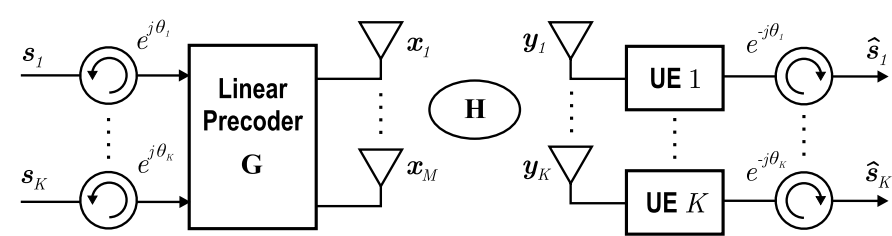

Fig. 1. Gyre Precoding block diagram.

\section{System Model And Linear Precoding PRELIMINARY}

Consider an MU-MIMO system where an $M$-antenna BS serves $K$ single-antenna non-cooperating UEs. In the downlink, signals are transmitted in frames of $N$ timeslots. The received baseband signals at timeslot $n=1, \ldots, N$ is given by the $K$-dimensional vector

$$
\mathbf{y}[n]=\mathbf{H} \mathbf{x}[n]+\mathbf{z}[n],
$$

where $\mathbf{x}[n]$ represents the $M$-dimensional vector of transmitted signals and $\mathbf{z}[n] \sim \mathcal{C N}\left(\mathbf{0}, \sigma_{z}^{2} \mathbf{I}_{K}\right)$ is the AWGN noise. The elements of the $K \times M$ matrix $\mathbf{H}$ denote the complex-valued, flat-fading channel coefficients from $m$-th BS antenna to the $k$-th UE, with $m \in[1, M]$ and $k \in[1, K]$. It is assumed that the BS has knowledge of $\mathbf{H}$, or an estimate thereof, which remains constant during the duration of the frame.

The input-output relation (1) can be written in matrix form for each frame as

$$
\mathbf{Y}=\mathbf{H} \mathbf{X}+\mathbf{Z}
$$

where $\mathbf{Y}=[\mathbf{y}[1], \mathbf{y}[2], \ldots, \mathbf{y}[N]], \mathbf{X}=[\mathbf{x}[1], \mathbf{x}[2], \ldots, \mathbf{x}[N]]$, and $\mathbf{Z}=[\mathbf{z}[1], \mathbf{z}[2], \ldots, \mathbf{z}[N]]$, with dimensions $K \times N, M \times$ $N$, and $K \times N$, respectively.

Furthermore, it is assumed that $\mathbf{X}=f(\mathbf{S})$, where $\mathbf{S}=$ $[\mathbf{s}[1], \mathbf{s}[1], \ldots \mathbf{s}[N]]$ with dimensions $K \times N$ are the data symbols to be transmitted and $f(\cdot)$ is the precoding function, which is chosen such that transmitted data can be recovered reliably at the UEs without the need for cooperation.

In the case of linear precoding, each transmitted vector $\mathbf{x}[n]$ is computed independently at each timeslot and is given by

$$
\mathbf{x}[n]=f(\mathbf{s}[n])=\frac{\mathbf{G} \mathbf{s}[n]}{\sqrt{\gamma}},
$$

where $\mathbf{G}$ is the linear precoder and

$$
\gamma=\frac{1}{N} \sum_{n=1}^{N}\|\mathbf{G} \mathbf{s}[n]\|^{2}
$$

is a scaling factor that imposes a unitary total transmitted power constraint, which is averaged over a frame.

In the case of ZF precoding $\mathbf{G}^{(\mathrm{ZF})}=\mathbf{H}^{H}\left(\mathbf{H H}^{H}\right)^{-1}$, where $(\cdot)^{H}$ is the conjugate transpose operator, and for the case of regularized, or MMSE precoding, $\mathbf{G}^{(\mathrm{MMSE})}=\mathbf{H}^{H}\left(\mathbf{H H}^{H}+\right.$ $\left.\sigma_{z}^{2} \mathbf{I}_{K}\right)^{-1}$. It is a well-known fact that ZF and MMSE precoding suffer from poor performance in ill-conditioned channels due to an SNR loss, mainly because $\left(\mathbf{H H}^{H}\right)^{-1}$ has a large eigenvalue that results in a large $\gamma[1]$.

\section{GyRE PRECODING}

The idea behind GP is to rotate the data symbols at the BS and perform a counter-rotation at the UEs, where the rotation angles for each layer $\theta_{k}, k=1, \ldots, K$ remain constant for the duration of the frame. We define $\boldsymbol{\Omega}=\left[\theta_{1}, \theta_{2}, \ldots, \theta_{K}\right]^{T}$ as the vector of the rotation angles, where $(\cdot)^{T}$ is the transpose operator. A block diagram of GP is depicted in Fig. 1.

When using GP, the transmitted signals can be written as

$$
\mathbf{x}[n]=f^{(\mathrm{GP})}(\mathbf{s}[n])=\frac{\mathbf{G} \mathbf{\Theta} \mathbf{s}[n]}{\sqrt{\gamma^{(\mathrm{GP})}}},
$$

where

$$
\boldsymbol{\Theta}=\operatorname{diag}\left(e^{j \boldsymbol{\Omega}}\right)=\left[\begin{array}{cccc}
e^{j \theta_{1}} & 0 & \cdots & 0 \\
0 & e^{j \theta_{2}} & \cdots & 0 \\
\vdots & \vdots & \ddots & \vdots \\
0 & 0 & \cdots & e^{j \theta_{K}}
\end{array}\right]
$$

with $\theta_{k} \in[0,2 \pi)$, and

$$
\begin{aligned}
\gamma^{(\mathrm{GP})} & =\frac{1}{N} \sum_{n=1}^{N}\|\mathbf{G} \boldsymbol{\Theta} \mathbf{s}[n]\|_{2}^{2} \\
& =\frac{1}{N}\|\mathbf{G} \boldsymbol{\Theta} \mathbf{S}\|_{\mathrm{F}}^{2}
\end{aligned}
$$

where $\|\cdot\|_{\mathrm{F}}$ is the Frobenius norm, and the step from (7) to (8) comes from the fact that the Frobenius norm is equivalent to the Euclidean norm when the underlying matrix is vectorized. The problem now becomes an optimization about how to find the data symbol rotations $\theta_{k}$ that minimize (8)

$$
\min _{\boldsymbol{\Theta}=\operatorname{diag}\left(e^{j \theta_{1}}, \ldots, e^{j \theta} K\right)} \gamma^{(\mathrm{GP})} \text {. }
$$

It can be noted that (9) has $K-1$ degrees of freedom, since a global phase $\phi$ can be extracted. This can be deduced from the right hand side of (8), since $\frac{1}{N}\left\|\mathbf{G} e^{j \phi} \boldsymbol{\Theta} \mathbf{S}\right\|_{\mathrm{F}}^{2}=\frac{1}{N}\|\mathbf{G} \Theta \mathbf{S}\|_{\mathrm{F}}^{2}$, for any $\phi \in \mathbb{R}$, due to the homogeneity property of norms.

The $\left\{\theta_{1}, \ldots, \theta_{K}\right\}$ angles need to be found jointly, but a closed-form solution of (9) is difficult to find. Therefore, we use an algorithm based on gradient descent [7] to iteratively solve (9) near-optimally. The gradient of (8) is given by

$$
\nabla \gamma^{(\mathrm{GP})}=\left[\begin{array}{c}
\frac{\partial}{\partial \theta_{1}} \gamma^{(\mathrm{GP})} \\
\vdots \\
\frac{\partial}{\partial \theta_{K}} \gamma^{(\mathrm{GP})}
\end{array}\right],
$$

where

$$
\begin{aligned}
\frac{\partial}{\partial \theta_{k}} \gamma^{(\mathrm{GP})} & =\frac{1}{N} \frac{\partial}{\partial \theta_{k}} \operatorname{tr}\left(\mathbf{S}^{H} \Theta^{H} \mathbf{G}^{H} G \mathbf{\Theta} \mathbf{S}\right) \\
& =\frac{1}{N} \operatorname{tr}\left(\mathbf{S}^{H} \frac{\partial \boldsymbol{\Theta}^{H}}{\partial \theta_{k}} \mathbf{G}^{H} \mathbf{G} \boldsymbol{\Theta} \mathbf{S}+\mathbf{S}^{H} \boldsymbol{\Theta}^{H} \mathbf{G}^{H} \mathbf{G} \frac{\partial \boldsymbol{\Theta}}{\partial \theta_{k}} \mathbf{S}\right) \\
& =\frac{2}{N} \operatorname{tr}\left(\mathfrak{R e}\left(\mathbf{S}^{H} \mathbf{\Theta}^{H} \mathbf{G}^{H} \mathbf{G} \frac{\partial \mathbf{\Theta}}{\partial \theta_{k}} \mathbf{S}\right)\right) \\
& =\frac{2}{N} \mathfrak{R e}\left(\operatorname{tr}\left(\mathbf{S S}^{H} \mathbf{\Theta}^{H} \mathbf{G}^{H} \mathbf{G} \frac{\partial \Theta}{\partial \theta_{k}}\right)\right)
\end{aligned}
$$

where $\operatorname{tr}(\cdot)$ is the trace operator. The steps in (11) and (12) are obtained by taking the partial derivative of (8) with respect to 
$\theta_{k}$, for $k \in[1, K]$. Eq. (13) is because the summands in (12) are Hermitian conjugates of each other, and because only the diagonal elements are considered due to the trace operation. Finally, (14) is due to the cyclic property of the trace function.

It should be noted that $\frac{\partial \boldsymbol{\Theta}}{\partial \theta_{k}}=\operatorname{diag}\left(0, \ldots, j e^{j \theta_{k}}, \ldots, 0\right)$, that is, it contains $j e^{j \theta_{k}}$ as the $k$-th element of the main diagonal, with all others being zero. Because of this, the trace operation in (14) contains only one non-zero value, and due to linearity, (10) can be expressed as

$$
\nabla \gamma^{(\mathrm{GP})}=\frac{2}{N} \mathfrak{R e}\left(\overline{\operatorname{diag}}\left(j \mathbf{S S}^{H} \boldsymbol{\Theta}^{H} \mathbf{G}^{H} \mathbf{G} \boldsymbol{\Theta}\right)\right)
$$

where the $\overline{\operatorname{diag}}(\mathbf{A})$ operator returns the elements of the main diagonal of $\mathbf{A}$ as a vector.

The gradient descent algorithm approximately solves (9) iteratively by updating the candidate $\Omega$ for step $t+1$ as

$$
\boldsymbol{\Omega}_{t+1}=\boldsymbol{\Omega}_{t}-\alpha_{t} \nabla \gamma_{t}^{(\mathrm{GP})}
$$

where $\alpha$ helps determines the step size. Smaller values of $\alpha$ result in a slow convergence, while large values of $\alpha$ may result in non-convergence. For GP, we found that for determining $\alpha$, the Barzilai-Borwein method [8] produces good results, and is given by

$$
\alpha_{t}=\frac{\left|\left(\boldsymbol{\Omega}_{t}-\boldsymbol{\Omega}_{t-1}\right)^{H}\left(\nabla \gamma_{t}^{(\mathrm{GP})}-\nabla \gamma_{t-1}^{(\mathrm{GP})}\right)\right|}{\left\|\nabla \gamma_{t}^{(\mathrm{GP})}-\nabla \gamma_{t-1}^{(\mathrm{GP})}\right\|_{2}^{2}} .
$$

The algorithm runs for a predetermined number of steps $T$ or when a stopping condition is met. For this work, we measure the norm of the gradient $\left|\nabla \gamma_{t}^{(\mathrm{GP})}\right|_{2}$ and stop whenever this value is below some small predetermined value $\delta$, which means a minimum has been nearly found. The pseudocode of the optimization procedure is presented in Algorithm 1. For our evaluations, $T=60$ and $\delta=10^{-8}$ were sufficient to produce results with comparable accuracy to those obtained from a brute-force search.

The data recovery process at each UE involves scaling and de-rotating the symbols. These operations can be written as

$$
\hat{s}_{k}[n]=\sqrt{\gamma^{(\mathrm{GP})}} e^{-j \theta_{k}} y_{k}[n],
$$

where $y_{k}[n]$ and $\hat{s}_{k}[n]$ are the received signal and the estimate of the transmitted data symbols, respectively, on the $k$-th UE.

From (18), it is clear that each UE needs to know their corresponding rotation angle $\theta_{k}$ and scaling factor $\gamma^{(\mathrm{GP})}$ in order for the data to be recovered reliably. This information can be broadcast from the BS to all UEs in the form of $K$ precoded pilots (one over each layer) sent during a single timeslot, which avoids a large signaling overhead. Pilots could be sent over more than one timeslot, with the intention of reducing the channel state information (CSI) error (including the phase errors). In either case, the pilot overhead would be $P /(N+P)$, where $P$ is the number of timeslots containing pilots. The provision of precoded pilots is included in some wireless standards, such as 3GPP's 5GNR [9]. It should be noted that for conventional linear precoding in the 5GNR standard, downlink precoded pilots are usually transmitted

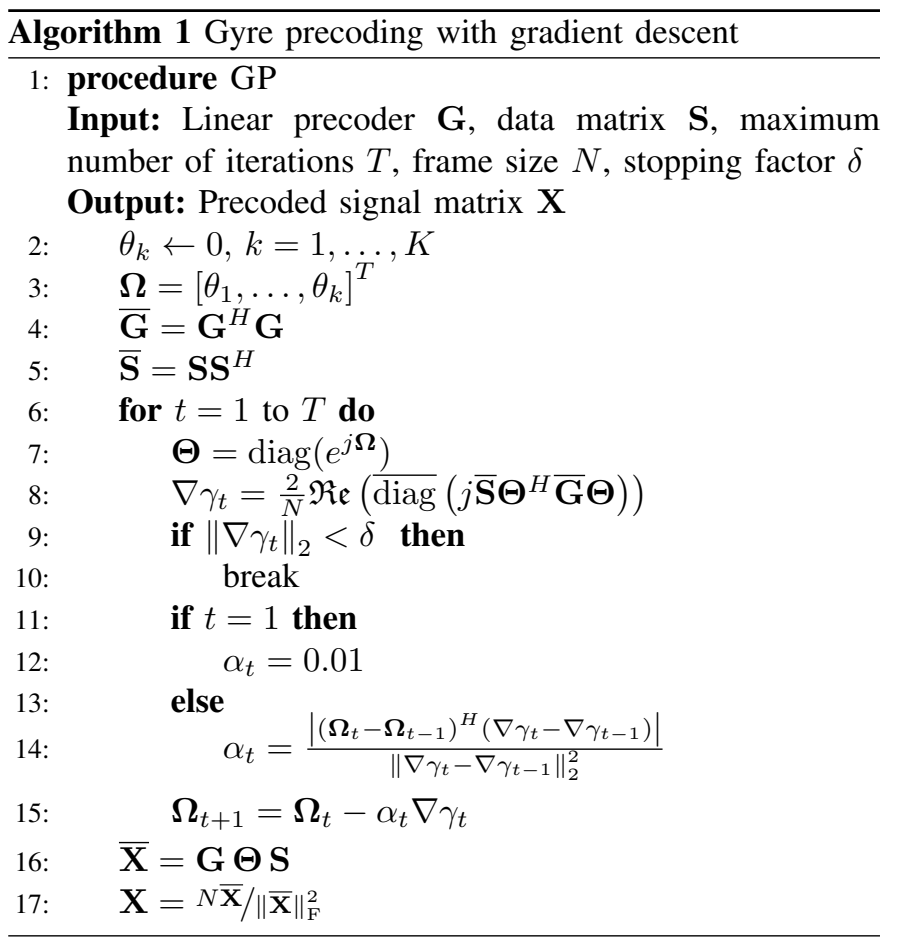

regardless. Therefore, in such cases, the use of GP would not incur in additional overhead.

Since GP is a linear technique, it retains many of the characteristics of conventional (non-GP) linear precoding. GP maintains the relative structure of the constellations. Therefore, the minimum distance between constellation points is preserved, and so is its noise immunity. For the same reason, the sensitivity of GP to phase jitter and CSI phase errors is comparable to that of conventional linear precoding. Furthermore, the peakto-average power ratio (PAPR) of the constellations and of the transmitted waveform (e.g., the orthogonal frequency-division multiplexing (OFDM) waveform used in 5GNR) is similar for GP and conventional linear precoding. These practical aspects of GP were validated by our evaluations, but are not presented in this letter due to space constraints.

\section{Performance aspects of GP}

\section{A. Complexity of $G P$}

The complexity of the optimization process can be deduced from Algorithm 1 and can be shown to be approximately

$$
K(K+1)(M+N) / 2+T\left(K^{2}+4 K\right)
$$

complex multiplications, where $T$ is the number of iterations. The $K(K+1)(M+N) / 2$ term reflects the number of operations to obtain the Hermitian matrices in steps 4 and 5. The bulk of the computations occur in step 8, with approximately $K^{2}+2 K$ operations per iteration, and steps 9 and 14 each require $K$ operations each per iteration. The GP optimization procedure is performed once per frame.

\section{B. GP dependence in channel condition and frame size}

GP offers an SNR gain over conventional linear techniques. The gain is dependent on two components: the channel matrix 


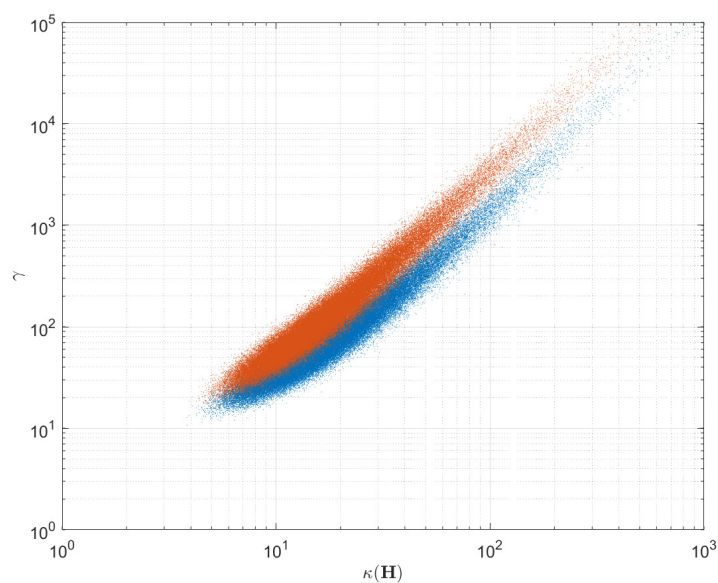

Fig. 2. Scatter plot of $\gamma^{(\mathrm{ZF})}$ (in red) and $\gamma^{(\mathrm{GP})}$ (in blue) vs. $\kappa(\mathbf{H})$ of $10^{5}$ realizations of an $8 \times 8$ system with Rayleigh fading, frame size $N=20$ and 16-QAM modulation.

TABLE I

AVERAGE NUMBER OF COMPLEX MULTIPLICATIONS PER FRAME FOR THE EVALUATED PRECODING METHODS.

\begin{tabular}{lrrrr}
\hline \multirow{2}{*}{ Precoding method } & \multicolumn{4}{c}{ MIMO size } \\
\cline { 2 - 5 } & $16 \times 4$ & $16 \times 8$ & $16 \times 12$ & $16 \times 16$ \\
\hline MMSE & 1,536 & 3,584 & 6,144 & 9,216 \\
MMSE-GP & 2,655 & 9,111 & 19,665 & 33,390 \\
CF-SLP & 5,536 & 14,412 & 26,774 & 43,218 \\
\hline
\end{tabular}

condition number $\kappa(\mathbf{H})$ and the frame size $N$. In GP, the frame size is defined as the symbol interval between the downlink precoded pilots.

The dependence of the performance of GP on the channel condition number can be observed in the scatter plot in Fig. 2 , where a comparison of the values of $\gamma$ obtained by GP and ZF precoding is plotted against the channel matrix condition number $\kappa(\mathbf{H})$. The results are for 10,000 realizations of $8 \times 8$ MIMO channel with Rayleigh fading and a frame size of 20 symbols. The figure shows that, in general, GP reduces $\gamma$ when compared to $\mathrm{ZF}$, and that the reduction is larger for higher values of $\kappa(\mathbf{H})$. This behavior can be explained by noting that as $\kappa(\mathbf{H})$ is reduced, $\mathbf{G}$ becomes more similar to a unitary matrix, which in the limit $(\kappa(\mathbf{H})=1)$ would make the norm in (7) invariant under $\Theta$.

Furthermore, the gains obtained by GP depend on the frame size. As shown in Section V (Fig. 4), GP offers high gains for short frame sizes, with the gain decreasing as the frame size grows. This is expected, since the rotation angles $\theta_{k}$ are common to all symbols in each layer of a frame. Therefore the $\gamma$ obtained by minimizing a single vector $(N=1)$ in (7) is smaller than the $\gamma$ that is obtained when optimizing over many vectors $(N \gg 1)$, with the gain of GP over linear precoding tends to diminish as $N \rightarrow \infty$. To obtain a high performance through the use of GP, the frame should be kept as short as possible. However, shorter frames also mean that the signaling overhead takes up a larger portion of the transmissions. Therefore, the frame size should be carefully chosen to maximize performance.

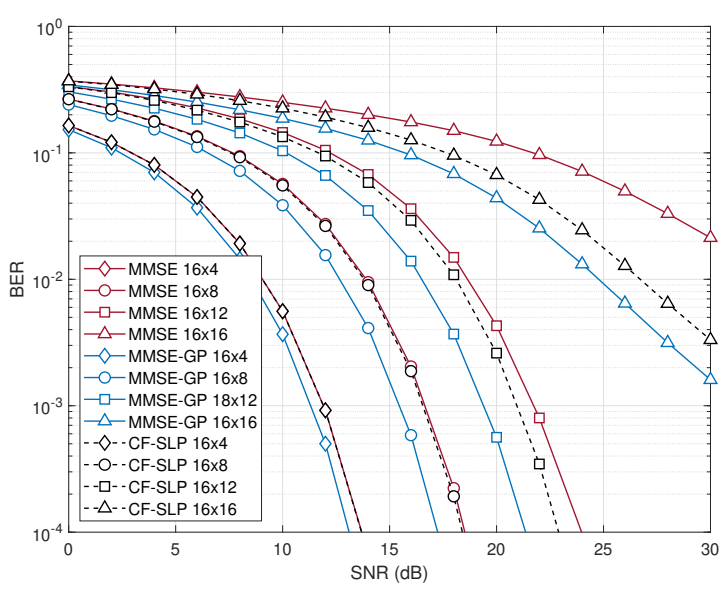

Fig. 3. BER plots of MMSE vs. MMSE-GP vs. CF-SLP with MMSE for an uncoded system with 16 BS antennas and $\{4,8,12,16\}$ UEs. Frame size is $N=20$ timeslots, the modulation used is 16-QAM.

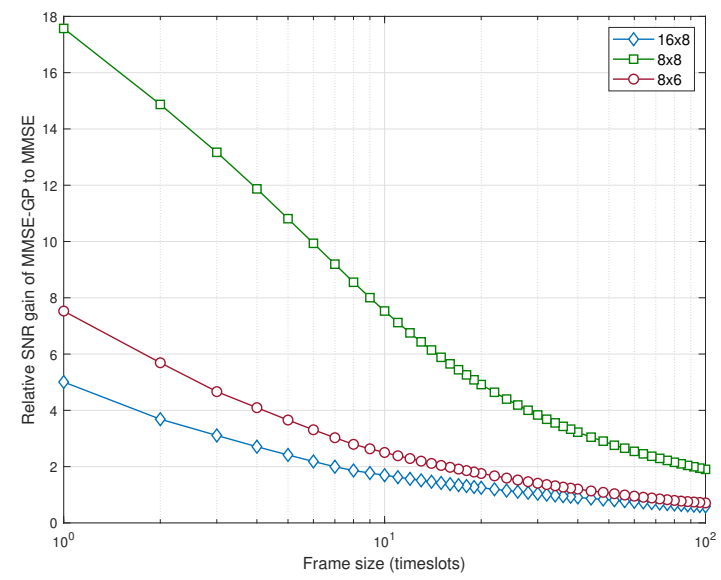

Fig. 4. Gain in SNR of MMSE-GP relative to MMSE vs. the frame size $N \in[1,100]$, at a BER of $10^{-2}$ Plots are for MIMO sizes $16 \times 8,8 \times 8$, and $8 \times 6$. Modulation is $16-\mathrm{QAM}$.

\section{Simulation Results}

This section presents results and insights obtained through computer simulations. Two experiments were conducted in two different scenarios.

Experiment 1: The objective of this test is to appraise GP and compare it to other precoding techniques. Particularly, we evaluate the performance of MMSE-GP to conventional MMSE and the closed-form suboptimal SLP (CF-SLP) approach presented in [5] (with an MMSE precoder); we chose this SLP method because it offers a reduction in complexity in relation to the highly-complex optimal SLP approach [5].

A block fading, frequency-flat Rayleigh channel is used, where the elements of $\mathbf{H}$ are i.i.d. zero-mean complex Gaussian random variables with unitary variance. It is assumed that $\mathbf{H}$ is perfectly known at the BS. The simulations use 16-QAM modulation with no forward error correction (FEC), and the SNR is defined as $1 / \sigma_{z}^{2}$.

Figure 3 shows bit error rate (BER) plots that compare MMSE-GP to MMSE precoding and to CF-SLP for an MUMIMO system with 16 BS antennas and $\{4,8,12,16\}$ UEs, 
and the frame size is $N=20$. From the figure, it can be observed that MMSE-GP outperforms the other two precoding techniques, with gains of $2 \mathrm{~dB}$ and $8 \mathrm{~dB}$ over CF-SLP and MMSE, respectively, in the $16 \times 16$ case at a BER of $10^{-2}$. It can also be observed that GP provides an SNR gain but not a diversity gain.

Furthermore, Table I shows the average complexity exhibited by the three precoding techniques. The table shows that MMSE offers the lowest complexity and that GP has lower complexity than CF-SLP, even though GP offers better error performance than the latter.

The error performance of GP depends on the frame size, as shown in Fig. 4. This figure shows the SNR gain of MMSEGP relative to MMSE, plotted against different frame sizes in the range $N \in[1,100]$, at a BER of $10^{-2}$. The plots are depicted for MIMO sizes of $16 \times 4,8 \times 8$, and $8 \times 6$. The results indicate that for short frames, large gains can be obtained, with decreasing gains as the frame number is increased. Moreover, the results in the figure asserts that GP can achieve large gains over conventional linear techniques for systems when the number of UEs is equal (or nearly equal) to the number of BS antennas.

Experiment 2: The aim of the second experiment was to evaluate GP in a massive-MIMO scenario in a 5GNR setting by using a 5GNR link-level simulator. This setup serves to validate the performance of GP in cases when the channel is well-conditioned and when practical channel estimation procedures are used in a standards-compliant setup. In this scenario, the base station ( $\mathrm{gNB}$ ) was equipped with a uniform linear array (ULA) with 64 cross-polarized, half wavelengthspaced antenna elements, with a directional radiation pattern based on [10, Sec. 7.3]. The UEs have a single omnidirectional antenna and are placed at an equal distance from the gNB, with a $5^{\circ}$ separation between them and with $5 \mathrm{~km} / \mathrm{h}$ mobility. The carrier frequency is $3.5 \mathrm{GHz}$, the RMS channel delay spread is $300 \mathrm{~ns}$, and the channel model used was the clustered delay line (CDL)-B [10]. Furthermore, time division duplexing (TDD) was used, and the precoder was calculated from the CSI estimates obtained from the uplink sounding reference signals (SRS), where perfect channel reciprocity was assumed. The downlink precoded pilots were carried over the demodulation reference signals (DM-RS) [9]. The configuration of the DMRS pilot structure was the following: mapping type 'A', type A position 2, maximum length 2 , additional position 1 , and configuration type 2 . The modulation is 16 -QAM with 0.5 -rate low-density parity-check (LDPC) coding, hybrid automatic repeat request (HARQ) was in use. The frames were defined as repeating $6 \times 7$ (subcarriers $\times$ symbols) blocks, which for the DM-RS setup that was used, corresponded to 30 data symbols $(N=30)$ and 12 pilots for each block.

The 5GNR scenario was evaluated for ZF and ZF-GP and for 4, 8, and 12 UEs. The experiment results are shown in Fig. 5 , where it can be observed that GP exhibits throughput gains over $\mathrm{ZF}(13 \%$ at $30 \mathrm{~dB}$ for the $64 \times 12$ case), and such gains increase with the number of UEs. This experiment confirms that GP offers improved performance over linear approaches in a massive-MIMO scenario with practical channel estimation procedures.

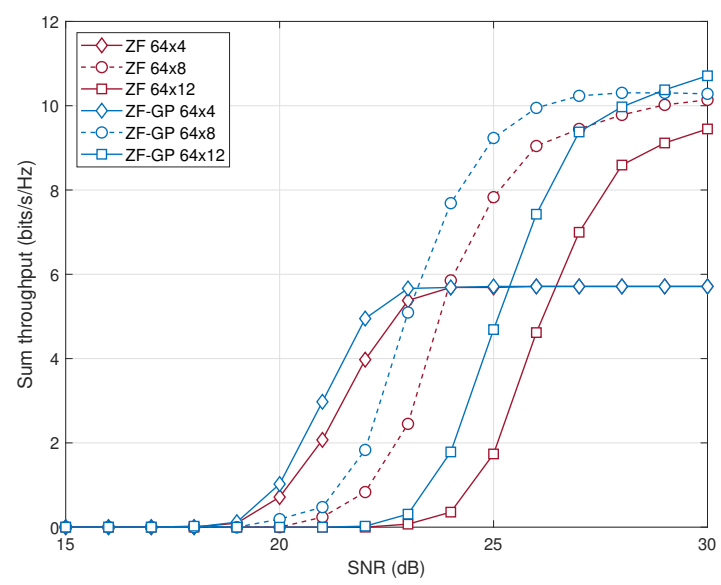

Fig. 5. Sum throughput of ZF vs ZF-GP for a massive-MIMO scenario with a 64-antenna gNB and 4, 8, and 12 UEs in a $5 \mathrm{G}$ NR environment with a CDL-B channel. The modulation is 16-QAM and coding is 0.5-rate LDPC.

\section{CONCLUSION}

We have presented Gyre Precoding, a simple, linear, and constellation-agnostic precoding technique that rotates the data symbols of each spatial layer to improve precoding performance. GP takes advantage of the short-term characteristics of the data symbols and achieves increasing SNR gains with shorter frame sizes. Furthermore, the proposed optimization algorithm exhibits low complexity and is processed once per frame. GP is compatible with conventional receiver processing procedures and wireless standards. The evaluation results show that GP can provide a significant power gain, while exhibiting comparable complexity, over conventional linear precoding and suboptimal SLP techniques. Moreover, it was verified that GP offers throughput gains in a massive-MIMO scenario with practical pilot schemes in a 5GNR standards-based setting.

\section{REFERENCES}

[1] C. B. Peel, B. M. Hochwald, and A. L. Swindlehurst, "A vector-perturbation technique for near-capacity multiantenna multiuser communication-part I: Channel inversion and regularization," IEEE Trans. Commun., vol. 53, no. 1, pp. 195-202, Jan. 2005.

[2] B. M. Hochwald, C. B. Peel, and A. L. Swindlehurst, "A vector-perturbation technique for near-capacity multiantenna multiuser communication-part II: Perturbation," IEEE Trans. Commun., vol. 53, no. 3, pp. 537-544, Mar. 2005.

[3] C. Husmann and K. Nikitopoulos, "Viper MIMO: Increasing large MIMO efficiency via practical vector-perturbation," in Proc. IEEE Global Commun. Conf. (GLOBECOM), Dec. 2018, pp. 1-6.

[4] C. Masouros and E. Alsusa, "Dynamic linear precoding for the exploitation of known interference in MIMO broadcast systems," IEEE Trans. Wireless Commun., vol. 8, no. 3, pp. 1396-1404, Mar. 2009.

[5] A. Haqiqatnejad, F. Kayhan, and B. Ottersten, "Power minimizer symbol-level precoding: A closed-form suboptimal solution," IEEE Signal Process. Lett., vol. 25, no. 11, pp. 1730-1734, Nov. 2018.

[6] M. Alodeh, S. Chatzinotas, and B. Ottersten, "Constructive multiuser interference in symbol level precoding for the MISO downlink channel," IEEE Trans. Signal Process., vol. 63, no. 9, pp. 2239-2252, May 2015.

[7] H. B. Curry, "The method of steepest descent for non-linear minimization problems," Quarterly of Applied Mathematics, vol. 2, no. 3, pp. 258-261, 1944.

[8] J. Barzilai and J. M. Borwein, "Two-point step size gradient methods," IMA journal of numerical analysis, vol. 8, no. 1, pp. 141-148, 1988.

[9] 5GNR; TS 38.211 Physical channels and modulation, 3GPP Std., Rev. 15.8.0, Dec. 2019.

[10] 5GNR; TR 38.901 Study on channel model for frequencies from 0.5 to $100 \mathrm{GHz}$, 3GPP Std., Rev. 15.0.0, Jun. 2018. 